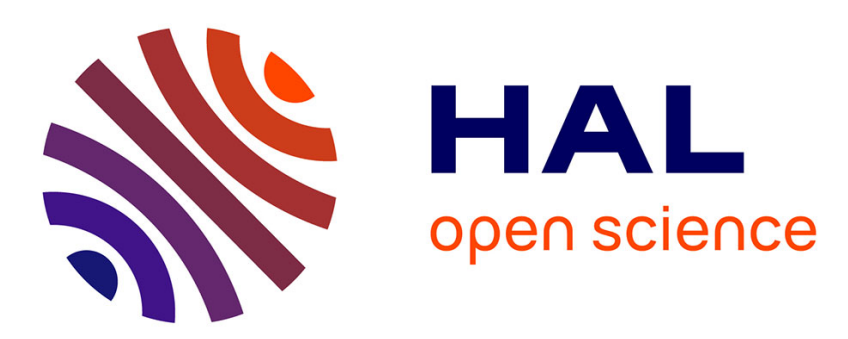

\title{
Distributions énergétique et angulaire de l'émission ionique secondaire. I. appareil expérimental Jean-François Hennequin
}

\section{To cite this version:}

Jean-François Hennequin. Distributions énergétique et angulaire de l'émission ionique secondaire. I. appareil expérimental. Revue de Physique Appliquée, 1966, 1 (4), pp.273-281. 10.1051/rphysap:0196600104027300 . jpa-00242733

\section{HAL Id: jpa-00242733 https://hal.science/jpa-00242733}

Submitted on 1 Jan 1966

HAL is a multi-disciplinary open access archive for the deposit and dissemination of scientific research documents, whether they are published or not. The documents may come from teaching and research institutions in France or abroad, or from public or private research centers.
L'archive ouverte pluridisciplinaire HAL, est destinée au dépôt et à la diffusion de documents scientifiques de niveau recherche, publiés ou non, émanant des établissements d'enseignement et de recherche français ou étrangers, des laboratoires publics ou privés. 


\title{
DISTRIBUTIONS ÉNERGÉTIQUE ET ANGULAIRE DE L'ÉMISSION IONIQUE SECONDAIRE I. APPAREIL EXPÉRIMENTAL.
}

\author{
Par Jean-François HENNEQUIN, \\ Lahoratoire de Physiciue des Solides, associé au C. N. R. S., \\ Faculté des Sciences, 91-Orsay.
}

\begin{abstract}
Résumé. - Nous avons entrepris l'étude expérimentale de l'émission ionique secondaire, et plus particulièrement des distributions énergétique et angulaire des ions secondaires émis lors du bcmbardement d'un échantillon par des ions primaires d'une énergie de quelques kiloélectrons-volts. Cette première partie du Mémoire est consacrée à la description de l'appareil que nous avons utilisé : principes de conception, réalisation mécanique et mise au point.
\end{abstract}

Abstract. - We have undertaken an experimental study of secondary ion emission, and in particular of the distribution in energy and angle of seconilary ions emitted during the bombardment of a sample by primary ions having an energy of a few kiloelectrons-:olts. The first part of the study is related to the description of the apparatus and its principles of nperation.

Introduction. - Alors que sont connus depuis longtemps les phénomènes de pulvérisation cathodique et d'émission électronique secondaire qui résultent du bombardement d'une cible par des ions primaires, ce n'est qu'assez récemment que différents auteurs [1 à 7] ont constaté la présence d'ions secondaires parmi les produits pulvérisés. Ces ions, formés essentiellement à partir des atomes de l'échantillon, sont donc pour la plupart caractéristiques de celui-ci. Ils ont ainsi pu être utilisés dans l'analyseur ionique de Castaing et Slodzian $[8,9]$ pour obtenir, au moyen d'un système optique approprié et avec une espèce déterminée d'ions, une image de la région bombardée, réalisant ainsi une analyse locale de l'échantillon avec un pouvoir séparateur de l'ordre du micron. D'autres spectromètres de masse $[10,11]$ ont été construits pour étudier la nature des ions secondaires, mais jusqu'à présent, en dehors des mesures de rendements ioniques de Beske [12], il n'existe que très peu de résultats expérimentaux concernant la distribution énergétique des ions émis $[4,13]$, et aucune donnée concernant leur répartition angulaire.

Nous présentons ici un appareil destiné à l'étude de la distribution énergétique et spatiale des ions secondaires. Après avoir donné les principes de conception et la réalisation mécanique de cet appareil, nous décrivons les expériences qui ont permis sa mise au point et la détermination de ses caractéristiques.

1) Paramètres expérimentaux. - Les différents paramètres qui interviennent dans l'émission d'ions secondaires, ainsi que les notations que nous utilisons pour chacun d'eux, sont précisés dans ce qui suit.
Les ions primaires $\mathrm{P}+$, de numéro atomique $Z_{1}$ et de masse atomique $M_{1}$, frappent l'échantillon avec une énergie $E_{1}$ sous un angle d'incidence $\theta$ ( fig. 1). Le rapport de l'intensité $I_{1}$ du faisceau primaire à la

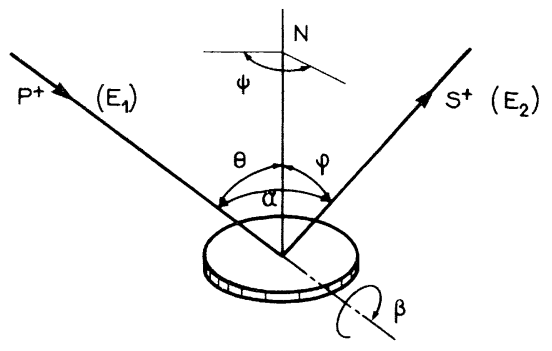

FIG. 1. - Paramètres expérimentaux.

surface $s$ bombardée donne la densité ionique moyenne $i_{1} \mathrm{du}$ bombardement. L'échantillon est le plus souvent un métal pur, de numéro atomique $Z_{\mathbf{2}}$ et de masse atomique $M_{2}$; mais ce peut être aussi un alliage, ou même un composé quelconque, pourvu qu'il soit peu volatil et possède une conductibilité électrique suffisante. Cet échantillon est placé, à la température $T$, dans une enceinte contenant un gaz dont la nature et la pression $p$ doivent être déterminées. En fait, lorsque la pression devient assez basse (inférieure à $10^{-6}$ torr), la nature du gaz résiduel n'intervient plus, du moins dans nos conditions de mesure.

Un ion secondaire $\mathrm{S}^{+}$, dont la nature est précisée par spectrométrie de masse, quitte l'échantillon avec une énergie $E_{2}$ dans une direction définie par les angles $\varphi$ et $\psi, \varphi$ étant l'angle d'émergence et $\psi$ l'angle azimutal (on prendra $\psi=0$ dans le plan 
d'incidence $\mathrm{du}$ côté du bombardement primaire). Bien entendu, dans le cas de l'étude d'un monocristal, l'orientation cristalline de la cible doit être connue. Dans un petit angle solide $\Delta \Omega$ autour de la direction $(\varphi, \psi)$, on mesure après filtrage en masse un courant secondaire $\Delta I_{2}$ et on peut introduire l'émission secondaire par unité d'angle solide :

$$
J_{2}(\varphi, \psi)=\frac{\mathrm{d} I_{2}}{\mathrm{~d} \Omega} \simeq \frac{\Delta I_{2}}{\Delta \Omega}
$$

comprenant tous les ions d'une espèce donnée S+ émis avec une énergie quelconque.

La distribution énergétique des ions émis dans la direction $(\varphi, \psi)$ peut être obtenue, soit directement par déflexion électrique ou magnétique, soit indirectement par une méthode de contre-champ. Dans ce dernier cas, pour une tension de contre-champ $E_{0}$, on recueille un courant :

$$
\Delta I\left(E_{0}\right)=\int_{E_{0}}^{\infty} e n(E) \mathrm{d} E
$$

$n(E)$ étant le nombre d'ions $\mathrm{S}+$ collectés par seconde dans la direction $(\varphi, \psi)$ à l'intérieur de l'angle solide $\Delta \Omega$, dont l'énergie initiale est comprise entre $E$ et $E+\mathrm{d} E$ ( $e$ est la charge électrique élémentaire). Le courant total est alors :

$$
\Delta I_{2}=\int_{0}^{\infty} e n(E) \mathrm{d} E .
$$

La courbe de distribution énergétique se déduit de la courbe de contre-champ par dérivation, car on a :

$$
n(E)=-\frac{1}{e} \frac{\mathrm{d}}{\mathrm{d} E_{0}} \Delta I\left(E_{0}\right)
$$

et l'énergie moyenne $\overline{E_{2}}$ des ions $\mathrm{S}^{+}$est :

$$
\overline{E_{2}}=\frac{\int_{0}^{\infty} E n(E) \mathrm{d} E}{\int_{0}^{\infty} n(E) \mathrm{d} E}=\frac{1}{\Delta I_{2}} \int_{0}^{\infty} \Delta I\left(E_{0}\right) \mathrm{d} E_{0}
$$

Cette énergie moyenne s'obtient donc en mesurant l'aire de la courbe de contre-champ (graphiquement ou par pesée) et en divisant cette aire par le courant mesuré en l'absence de contre-champ. On peut d'ailleurs, pour permettre une comparaison plus aisée des courbes de contre-champ, prendre ce courant total $\Delta I_{2}$ comme unité et porter sur le graphique la quantité normalisée : $\Delta I\left(E_{0}\right) / \Delta I_{2} ;$ l'énergie moyenne $\overline{E_{2}}$ est alors numériquement égale à l'aire de la courbe (fig. 2).

Remarquons que le calcul de $\overline{E_{2}}$ suppose que le courant avec contre-champ $\Delta I\left(E_{0}\right)$ tend vers zéro, pour $E_{0}$ tendant vers l'infini, plus rapidement que 1/E. D'autre part, il est difficilement concevable

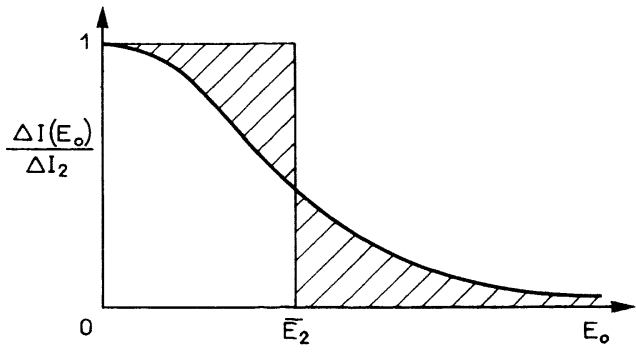

Fig. 2. - Détermination de l'énergie moyene $\overline{E_{2}}$.

qu'une particule secondaire puisse être éjectée avec une énergie initiale $E_{2}$ supérieure à l'énergie $E_{1}$ de l'ion primaire; la limite supérieure des intégrales devrait donc être au plus $E_{1}$ et non l'infini. En fait, les énergies $E_{2}$ sont toujours bien inférieures à $E_{1}$ et aucune modification sensible de la valeur de $\overline{E_{2}}$ ne peut apparaître dans ce calcul.

L'émission totale $I_{2}$ d'une espèce donnée d'ions secondaires s'obtient par intégration, sur toutes les directions $(\varphi, \psi)$ possibles d'un demi-espace, de l'émission $J_{2}(\varphi, \psi)$, soit :

$$
I_{2}\left(\mathrm{~S}^{+}\right)=\iint J_{2}(\varphi, \psi) \mathrm{d} \Omega=\iint \frac{\Delta I_{2}}{\Delta \Omega} \sin \varphi \mathrm{d} \varphi \mathrm{d} \psi .
$$

Ce calcul se simplifie notablement dans le cas du bombardement à incidence normale $(\theta=0)$ d'un polycristal, car la symétrie de révolution implique que la densité $J_{2}$ ne dépende plus que de $\varphi$, et done :

$$
\begin{aligned}
I_{2}\left(\mathrm{~S}^{+}\right)=2 \pi \int_{0}^{\pi / 2} \frac{\Delta I_{2}}{\Delta \Omega} & \sin \varphi \mathrm{d} \varphi \\
& \simeq 2 \pi \frac{\Delta \varphi}{\Delta \Omega} \sum_{0}^{\pi / 2} \Delta I_{2} \sin \varphi
\end{aligned}
$$

( $\Delta \varphi$ étant le pas choisi pour le calcul de la somme). Le rendement $K_{\mathrm{M}}^{\mathrm{P}^{+}}\left(\mathrm{S}^{+}\right)$de l'émission en ions secondaires $\mathrm{S}^{+} \mathrm{du}$ métal $\mathrm{M}$ bombardé par des ions primaires $\mathrm{P}+$ (souvent noté plus simplement $K$ ) s'obtient enfin par le rapport : $K=I_{2}(\mathrm{~S}+) / I_{1}$.

Les ions secondaires sont parfois collectés [9] en appliquant un champ électrique intense à la surface de l'objet. Cette méthode, indispensable dans l'analyseur ionique si l'on veut obtenir un pouvoir séparateur satisfaisant sur l'image, présente en outre l'avantage d'accroître considérablement le courant secondaire recueilli, puisque même des ions émis avec un angle d'émergence assez grand seront encore collectés. Mais il s'introduit simultanément une discrimination selon l'énergie initiale des ions, qui rend aléatoire toute comparaison quantitative ultérieure. Un tel procédé est donc limité à l'étude de la nature des ions secondaires, où l'obtention de courants ioniques élevés est de première importance. Pour conserver les directions initiales d'éjection des ions, 
il est indispensable que l'échantillon soit placé dans une région de champ électrique nul.

2) Conception de 1'appareil. - La solution idéale pour obtenir un faisceau d'ions primaires d'intensité élevée, de nature et d'énergie connues, consiste à associer une source à arc à cathode chaude avec un déflecteur magnétique. Les sources à arc à cathode chaude ont en effet une très faible dispersion énergétique (quelques électrons-volts), conduisent facilement à des courants de plusieurs dizaines de microampères, voire du milliampère, et permettent l'ionisation d'un grand nombre d'éléments à partir du corps simple correspondant ou d'un composé convenable [14, 15]. La déflexion magnétique ultérieure permet de préciser la nature des ions primaires obtenus, tout en éliminant du faisceau les atomes neutres rapides provenant d'une neutralisation partielle inévitable dans la région de l'extraction des ions, où la pression est encore relativement élevée.

Ce montage est cependant de réalisation et de maintenance assez compliquée et nous avons cherché une solution plus simple. A condition de se limiter aux ions de gaz rares, il est possible, au moyen d'une source à excitation haute fréquence $[16,17]$, d'obtenir aisément un faisceau ionique assez intense ne contenant pratiquement que des ions simples chargés une seule fois, et dont la dispersion énergétique à $10 \mathrm{kV}$ d'accélération n'excède pas $500 \mathrm{eV}$. Dans ces conditions, le filtrage magnétique n'est plus nécessaire et nous avons utilisé une déflexion électrique, de réalisation beaucoup plus simple, pour éliminer les atomes neutres rapides et focaliser le faisceau primaire en un point convenable de l'échantillon, tout en limitant à une centaine d'électrons-volts la dispersion énergétique des ions primaires de $10 \mathrm{keV}$. L'ionisation d'autres gaz (hydrogène, azote, oxygène, chlore...) est d'ailleurs elle aussi possible dans une source haute fréquence, mais alors la déflexion magnétique devient indispensable pour trier les ions moléculaires et les ions plusieurs fois chargés. Nous avons renoncé à réaliser une telle déflexion en raison de l'encombrement excessif de l'électro-aimant qu'il aurait fallu utiliser pour filtrer ces ions relativement rapides.

$\mathrm{La}$ variation des trois paramètres angulaires $(\theta, \varphi, \psi)$ pose un problème mécanique délicat ; un seul des trois systèmes : source d'ions-échantillonspectromètre de masse, peut rester fixe. S'il est facile de faire tourner ou d'incliner un échantillon, il est beaucoup plus difficile de déplacer sous vide une source d'ions ou un spectromètre de masse, en raison de l'encombrement de ces montages et des connexions électriques à assurer. Nous avons choisi de maintenir fixe la source d'ions, la déflexion magnétique des ions secondaires étant alors assurée, comme nous le verrons plus loin, par un aimant permanent, ce qui réduit considérablement l'encom- brement du spectromètre de masse et permet de le rendre mobile à l'intérieur de l'enceinte à vide. Toutes ces nécessités mécaniques nous conduisent aux trois mouvements suivants ( $f$ ig. 1 ) :

- inclinaison de l'échantillon autour de l'un de ses diamètres (angle $\theta$ ),

- rotation du spectromètre de masse autour d'un axe vertical perpendiculaire à la direction du faisceau primaire (angle $\alpha$ ),

- rotation de l'échantillon autour d'un axe horizontal, confondu avec la direction du faisceau primaire (angle $\beta$ ).

On obtient les angles liés à l'échantillon $(\theta, \phi, \psi)$ à partir des trois angles expérimentaux $(\theta, \alpha, \beta)$ par l'intermédiaire des formules de la trigonométrie sphérique :

$$
\left\{\begin{array}{l}
\cos \varphi=\cos \alpha \cos \theta+\sin \alpha \sin \theta \cos \beta \\
\cos \psi=\frac{\cos \alpha \sin \theta-\sin \alpha \cos \theta \cos \beta}{\sin \varphi}
\end{array}\right.
$$

Inversement, si l'on se donne les trois angles $(\theta, \varphi, \psi)$, c'est-à-dire les conditions particulières d'un bombardement, on déduit les angles expérimentaux $(\theta, \alpha, \beta)$ à afficher, des formules :

$$
\left\{\begin{array}{l}
\cos \alpha=\cos \theta \cos \varphi+\sin \theta \sin \varphi \cos \psi \\
\cos \beta=\frac{\sin \theta \cos \varphi-\cos \theta \sin \varphi \cos \psi}{\sin \alpha} .
\end{array}\right.
$$

En fait, l'étude de l'émission est le plus souvent conduite selon l'un des deux cas particuliers suivants :

— bombardement à incidence normale : $\theta=0$; $\alpha=\varphi ; \beta=\psi$,

— étude dans le plan d'incidence : $\psi=0$ (ou $\pi)$; $\alpha=\varphi \pm \theta ; \beta=0$ (ou $\pi$ ).

La variation simultanée sous vide de ces trois angles $(\theta, \alpha, \beta)$ serait de réalisation mécanique difficile ; en fait l'angle $\theta$ est fixé avant montage et ne peut être modifié qu'après ouverture de l'appareil.

Nous avons renoncé aussi à faire varier la température de l'échantillon. Il serait certes possible de chauffer par rayonnement, au moyen d'un petit four à résistance, l'extrémité du bras porte-échantillon ; mais nous avons préféré monter à cet endroit une glissière en queue d'aronde afin de pouvoir bombarder plusieurs zones successives sur le même échantillon. Signalons aussi à ce propos que, dans nos conditions de mesures, l'échauffement de la cible sous l'impact des ions primaires reste insignifiant.

Le dispositif d'étude des ions secondaires doit réaliser la séparation d'ions de masses différentes dans une large bande d'énergies : un spectromètre de masse à double focalisation semblerait donc s'imposer [10]. En fait, un tel appareil est très encombrant et il est hors de question de le déplacer sous vide; il semble préférable de séparer l'étude de la 
nature des ions secondaires (qui sera confiée à un spectromètre de masse avec champ électrique extracteur) et l'étude de leur distribution énergétique et spatiale. En effet, une proportion très notable des ions secondaires issus d'un métal $M$ est constituée d'ions simples du type $\mathbf{M}+[2,9]$. Si donc on est certain qu'il n'existe pas d'ions de masses voisines dans l'émission du métal M, un filtrage magnétique rudimentaire est alors suffisant, et de plus n'introduit qu'une faible discrimination énergétique parmi les ions collectés, en raison de la faible dispersion du montage. Remarquons toutefois qu'à l'exception du lithium, les différents isotopes d'un même élément seront alors collectés simultanément.

Un simple aimant permanent peut donc être utilisé; l'angle de déflexion $\Phi$ des ions $M+$ est fixé avant montage par calage du cylindre de Faraday de collection, les retouches éventuelles étant introduites en faisant varier légèrement la tension d'accélération $U_{2}$ des ions secondaires. Si $\rho$ est le rayon des pièces polaires de l'aimant (compte tenu du champ de fuite) et $B$ l'induction réalisée dans l'entrefer, l'angle de déflexion $\Phi$ est tel que :

$$
\begin{aligned}
\operatorname{tg} \frac{\Phi}{2} & =\rho B \sqrt{\frac{e}{2 m U_{2}}}=\rho B \sqrt{\frac{F}{2 M_{2} U_{2}}} \\
& (F: \text { Faraday })
\end{aligned}
$$

et la dispersion énergétique du prisme magnétique telle que :

$$
\frac{\Delta \Phi}{\Delta U_{2}}=-\frac{1}{2} \frac{\sin \Phi}{U_{2}}
$$

Il y a donc tout intérêt à travailler avec une tension d'accélération élevée ; on est toutefois limité dans ce sens par l'induction maximale qu'il est possible d'obtenir dans l'aimant permanent, la largeur de l'entrefer étant déterminée par le diamètre du faisceau ionique.
La distribution énergétique des ions secondaires pourrait s'obtenir directement par déflexion électrostatique, après sélection des ions étudiés par filtrage magnétique. En fait, avec les faisceaux ioniques relativement larges et ouverts qu'il faut utiliser pour recueillir un courant secondaire raisonnable, la résolution d'une telle déflexion électrique est très médiocre [181 et il semble préférable d'utiliser une méthode de contre-champ. L'application d'une tension de contre-champ sur le cylindre de Faraday lui-même est usuelle, mais conduit souvent à des résultats d'interprétation délicate en raison des électrons secondaires qui sont simultanément collectés. Nous avons préféré placer une grille très fine sur le trajet des ions secondaires, avant l'électrode d'accélération, mais en dehors de la région de champ nul qui entoure l'échantillon; il sera, bien entendu, tenu compte de la transparence de cette grille pour obtenir le courant ionique vrai. La détermination de la distribution énergétique des ions secondaires à partir de la courbe de contre-champ a déjà été étudiée (voir paragraphe 1).

3) Réalisation mécanique. - La figure 3 montre le schéma de principe de l'appareil conçu selon les considérations précédentes. Le dispositif d'obtention du faisceau primaire, situé dans un plan vertical, figure à gauche, tandis que le dispositif d'étude des ions secondaires, mobile dans un plan horizontal, est présenté à droite.

Le faisceau d'ions primaires est obtenu à partir d'une source à excitation haute fréquence, constituée d'une ampoule de pyrex à l'intérieur de laquelle le gaz à ioniser est introduit par un robinet à aiguille. Le plasma ainsi créé est porté à une haute tension positive $U_{1}$ qui peut varier de 2 à $10 \mathrm{kV}$; l'extraction des ions se fait entre la limite inférieure de ce plasma et une électrode d'extraction en duralumin, entourée

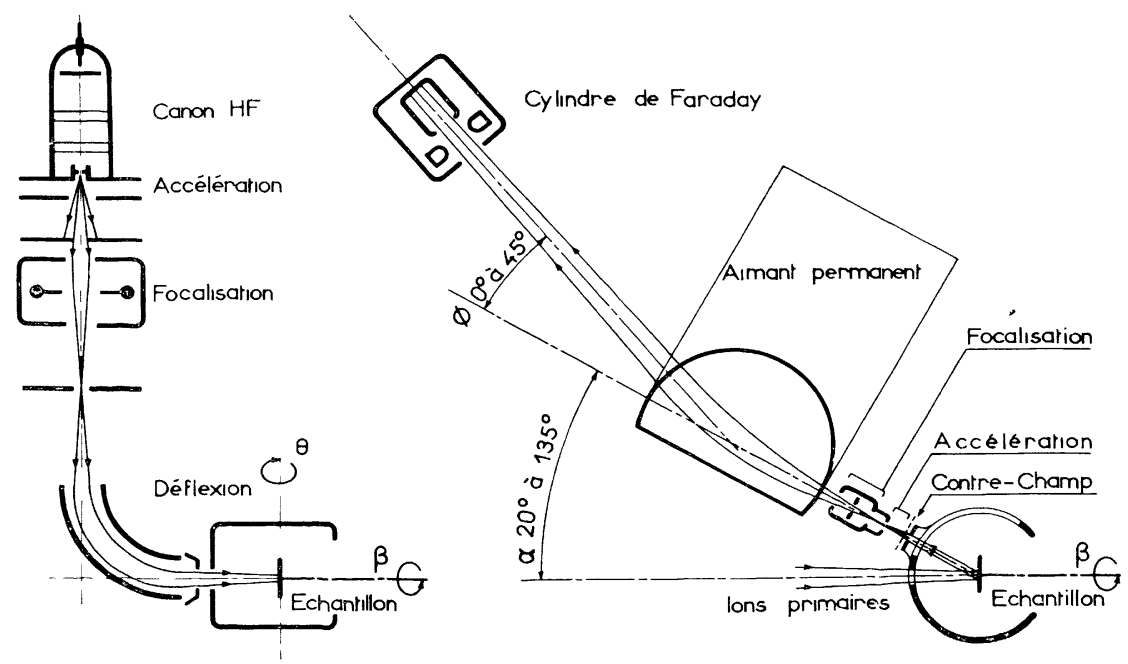

FIg. 3. - Schéma de principe de l'appareil. 
d'un cylindre de quartz qui, en retenant des charges positives, repousse les ions et joue ainsi le rôle d'un wehnelt. Un condenseur focalise ensuite les ions primaires à l'entrée du déflecteur électrostatique sphérique. A ce niveau, une pompe à diffusion d'huile indépendante assure l'évacuation du gaz provenant du canon, tandis qu'un diaphragme de $1,2 \mathrm{~mm}$ de diamètre isole toute cette partie de l'appareil de l'enceinte à vide poussé ; l'ensemble canon-condensateur est centrable mécaniquement par rapport à ce diaphragme d'isolement. Le déflecteur focalise les ions primaires sur l'échantillon en un spot de surface voisine du millimètre carré, l'intensité du courant primaire variant de 1 à $10 \mu \mathrm{A}$ selon la nature de ces ions et leur tension d'accélération. L'échantillon et le cylindre qui l'entoure sont portés à un potentiel positif $U_{2}$ de l'ordre de $2 \mathrm{kV}$ destiné à accélérer les ions secondaires : l'énergie $E_{1}$ des ions primaires au moment où ils frappent l'objet a donc pour valeur :

$$
E_{1}=e\left(U_{1}-U_{2}\right)
$$

L'échantillon se présente sous la forme d'une pastille de $10 \mathrm{~mm}$ de diamètre et d'environ $1,5 \mathrm{~mm}$ d'épaisseur; on peut d'ailleurs monter une tôle plus mince, en disposant par derrière une rondelle métallique quelconque. Il est possible aussi de confectionner une telle pastille à partir d'une poudre, par compression à la presse hydraulique, avec adjonction éventuelle de cuivre en poudre, pour accroître la conductibilité électrique et donner une meilleure cohésion mécanique. La surface des échantillons métalliques est polie mécaniquement, puis électrolytiquement; en fait si le polissage électrolytique, précédé au besoin d'un décapage chimique, est indispensable pour les monocristaux, il ne s'avère pas nécessaire pour les échantillons polycristallins : après quelques minutes de bombardement, le courant ionique se stabilise à la même valeur quel que soit le type de polissage subi par la cible. La couche superficielle d'oxyde étant alors pulvérisée, la surface décapée présente sans doute un état microscopique indépendant de son état initial.

Le montage permet aussi l'étude du dépôt formé par les atomes neutres de la cible, arrachés par pulvérisation cathodique; on dispose à cet effet sur la face interne du cylindre qui entoure l'échantillon, un morceau de film photographique $35 \mathrm{~mm}$, dont la gélatine a été enlevée à l'eau de Javel. Un trou est évidemment percé dans ce film pour laisser le passage au faisceau primaire. Le support d'acétate de cellulose est assez transparent pour autoriser le relevé densitométrique de la couche métallique ainsi déposée. Dans tous les cas, l'intensité du courant primaire est mesurée par un microampèremètre disposé dans la connexion d'amenée de tension à l'échantillon. La disposition de ce dernier à l'intérieur d'une chambre, à laquelle il est élec- triquement relié, constitue un véritable cylindre de Faraday, et, généralement, aucune correction d'émission électronique secondaire n'est nécessaire.

L'échantillon étant placé à l'intérieur d'un cylindre porté au même potentiel que lui, les directions d'émission des ions secondaires sont conservées. Le faisceau primaire pénètre par un orifice percé dans ce cylindre, tandis qu'une fente permet la sortie des ions secondaires; une large échancrure située derrière la cible assure un pompage efficace de cette région et laisse le passage au bras porteéchantillon. Une première électrode, portée par un bras tournant et percée d'un trou circulaire de 1,3 $\mathrm{mm}$ de diamètre, est disposée contre le cylindre et reliée électriquement à celui-ci par un contact glissant; l'ouverture du faisceau secondaire est ainsi fixée à 1/30 de radian, ce qui correspond à un angle solide de collection $\Delta \Omega=3,5 \times 10^{-3}$ stéradians. A la suite est placée une grille de cuivre au pas de $25 \mu \mathrm{m}$ et de transparence $50 \%$; il lui est appliquée une tension supérieure à celle de l'échantillon. On introduit ainsi une barrière de potentiel que seuls les ions d'énergie plus élevée pourront franchir ; cette méthode de contre-champ conduit à la distribution énergétique des ions secondaires (voir paragraphe 1). Le faisceau est ensuite accéléré, puis focalisé par une lentille à trois électrodes (le projecteur), et, après rotation dans le champ magnétique créé par l'aimant permanent, pénètre dans un cylindre de Faraday soigneusement blindé et précédé d'une électrode portée à une tension légèrement négative (-100 V) destinée à éliminer l'effet parasite des électrons secondaires.

Tout ce dispositif optique est porté par un bras tournant qui permet de balayer sous vide les directions comprises entre $20^{\circ}$ et $135^{\circ}$ par rapport à l'axe du faisceau primaire (angle $\alpha$ ). Par rotation du bras porte-échantillon, il est possible aussi de faire tourner la cible autour du même axe (angle $\beta$ ) : toutes les directions de l'espace peuvent donc être engendrées (voir paragraphe 2 et fig. 1). Plusieurs points d'impacts du faisceau primaire sont obtenus par déplacement latéral du bras porte-échantillon; cette manipulation, comme les deux précédentes, s'effectue sous vide, depuis l'extérieur de l'enceinte, au moyen de passages tournants à membrane flexible. Par contre, ce n'est qu'après ouverture de l'appareil que peuvent être modifiés l'incidence (angle $\theta$ ) du faisceau primaire et le calage (angle $\Phi$ ) du cylindre de Faraday. Cet angle $\Phi$ est calculé en fonction des ions que l'on désire étudier, de manière à travailler avec une tension d'accélération $U_{2}$ voisine de $2 \mathrm{kV}$. L'isolement des entrées de tension étant de $5 \mathrm{kV}$, on peut ainsi étudier les ions de masse double du type $\mathrm{M}_{2}^{+}$(avec $U_{2}=1 \mathrm{kV}$ ) et les ions deux fois chargés du type $\mathrm{M}++$ (avec $U_{2}=4 \mathrm{kV}$ ), le réglage étant fait pour les ions simples du type $\mathrm{M}^{+}$. Il n'est pas possible cependant d'en tirer des relations quantitatives, sans modifier 
simultanément la tension d'accélération $U_{1}$ des ions primaires, de manière à maintenir constante leur énergie $E_{1}=e\left(U_{1}-U_{2}\right)$ à l'arrivée sur l'échantillon.

Le courant ionique est mesuré au moyen d'un picoampèremètre Lemouzy, le signal étant "sorti * par une connexion très soigneusement blindée. Le montage d'un multiplicateur d'électrons accroîtrait certes la sensibilité du dispositif, mais la place disponible à l'intérieur de l'enceinte ne permet pas l'installation d'un tel appareil. Les courants ioniques d'autre part, sont, en général, nettement supérieurs au picoampère: les difficultés de mesure (dérive du zéro notamment) apparaissent donc seulement aux fortes tensions de contre-champ, où elles se superposent aux limitations introduites par le dispositif de filtrage (voir paragraphe 4).

$\mathrm{La}$ nature et la pression du gaz qui entoure l'échantillon modifient l'émission secondaire de celui-ci ; ce n'est qu'à une pression très basse que l'émission devient indépendante du gaz résiduel. Il convient donc de réaliser un vide poussé au voisinage de la cible, et ce malgré le voisinage d'une source d'ions à l'intérieur de laquelle la pression du gaz atteint $10^{-3}$ torr. Seul un pompage différentiel permet de satisfaire à cette exigence : une pompe auxiliaire de $300 \mathrm{l} / \mathrm{s}$, à diffusion d'huile et équipée d'un baffle à eau, assure le pompage de la région comprise entre l'extraction des ions primaires et le diaphragme d'isolement à l'entrée du déflecteur électrostatique; elle maintient dans cette région une pression de l'ordre de $10^{-5}$ torr quand le canon à ions est en fonctionnement. Comme le diamètre du diaphragme d'isolement est de 1,2 $\mathrm{mm}$, le débit du gaz résiduel à travers ce trou est voisin de $0,2 \mathrm{l} / \mathrm{s}$ (le calcul est fait pour de l'argon). La pompe principale, elle aussi à diffusion d'huile, possède une vitesse de pompage propre de $300 \mathrm{l} / \mathrm{s}$; en raison de la présence d'un piège à azote liquide, destiné à réduire la rétrodiffusion de l'huile de la pompe à diffusion vers l'enceinte, cette vitesse est réduite à $150 \mathrm{l} / \mathrm{s}$, mais reste encore très grande devant le débit du gaz à travers le trou du diaphragme d'isolement. Il en résulte que la pression dans l'enceinte n'est pratiquement limitée que par le dégazage des parois, et doit être à peu près insensible à la variation de la pression du gaz dans le canon, selon que celui-ci est alimenté ou non.

Ce point est confirmé par l'expérience, et une jauge à ionisation du type Bayard-Alpert montée sur l'enceinte indique une pression de l'ordre de $2 \times 10^{-7}$ torr pendant les mesures. Un tel vide, qui nécessite d'ailleurs près de 5 heures de pompage, est obtenu par polissage et nettoyage soigné de toutes les pièces, et par utilisation systématique de joints métalliques en indium dans le montage de l'enceinte, à l'exception du couvercle supérieur où un joint «viton " est conservé pour permettre un démontage plus aisé (le canon est lui aussi équipé en joints "viton »). Précisons aussi que l'appareil est le moins souvent possible ouvert à l'air libre ; quand cela est nécessaire, c'est de l'argon qui est introduit à l'intérieur et non de l'air, en vue de réduire au minimum les traces d'humidité, qui accroissent notablement les temps de pompage. Par étuvage prolongé, il serait en principe possible d'abaisser encore la pression du gaz résiduel, mais la réalisation de l'appareil en alliages légers et en laiton rend une telle opération périlleuse ; par ailleurs, comme le montre le calcul suivant, un vide plus poussé ne semble pas vraiment indispensable.

Il est possible en effet d'estimer l'action du gaz résiduel en admettant que son influence disparaît quand le nombre de particules arrachées à l'échantillon est très supérieur au nombre de molécules du gaz résiduel qui arrivent sur celui-ci, et qui d'ailleurs ne s'y fixent que durant un temps très court (tout au moins tant qu'il ne se produit pas de réaction chimique à la surface : fixation d'oxygène ou formation d'une couche de contamination par polymérisation des molécules lourdes provenant de l'huile des pompes à vide). Si la pression du gaz résiduel est $p$ et sa masse molaire $M$, le nombre $\nu$ de molécules qui heurtent la surface $s$ de l'échantillon pendant une seconde, à la température $T$, est :

$$
\nu=N p s / \sqrt{2 \pi M R T}
$$

( $N$ : nombre d'Avogadro, $R$ : constante des gaz parfaits).

Dans le même temps, le nombre d'ions primaires qui frappent la même surface est : $s i_{1} / e, i_{1}$ étant la densité ionique du faisceau primaire et $e$ la charge électrique élémentaire. Si $S$ est le rendement de pulvérisation, dans les conditions du bombardement, de la substance dont est constitué l'échantillon, on doit réaliser la condition :

$$
\frac{S i_{1}}{\overline{F p}} \sqrt{ } 2 \overline{\pi M R T} \gg 1 \quad(F: \text { Faraday }) .
$$

Les rendements de pulvérisation $S$ sont généralement quelque peu supérieurs à l'unité ; la valeur 1 nous fournit donc une estimation trop sévère. Si l'on mesure la pression en torrs et la densité ionique en $\mu \mathrm{A} / \mathrm{mm}^{2}$, et si l'on suppose que le gaz résiduel est de l'argon, on aboutit à la condition numérique : $p \ll 2 \times 10^{-6} i_{1}$.

Avec les densités ioniques que nous utilisons (de 1 à $10 \mu \mathrm{A} / \mathrm{mm}^{2}$ ) il y a donc de 10 à 100 fois plus d'ions incidents que de molécules du gaz résiduel à venir heurter l'échantillon. On conçoit alors que la nature de ce gaz résiduel n'ait plus qu'une incidence très faible sur l'émission secondaire. Ajoutons que nous n'observons aucune trace de contamination au voisinage de la tache décapée et que d'autre part l'introduction d'un gaz inerte dans 
l'enceinte n'apporte aucune modification de l'émission (les mesures ont été conduites jusqu'à une pression de $2 \times 10^{-4}$ torr). L'oxygène par contre provoque le plus souvent un accroissement considérable de l'émission, dû vraisemblablement à la formation d'une liaison chimique avec le métal de la cible.

4) Mise au point de l'appareil, - Nous avons d'abord ajusté les tensions électriques appliquées aux deux électrodes du déflecteur électrostatique de façon à rendre maximal le courant tombant sur l'échantillon, tout en le concentrant en un spot aussi rond que possible. Par suite d'un faible balayage du faisceau à la sortie du déflecteur (en raison sans doute de légères fluctuations dans le plasma du canon), la région pulvérisée reste cependant légèrement allongée dans le sens vertical ; elle comporte une partie centrale fortement décapée, de surface voisine du millimètre carré, entourée d'un halo beaucoup plus faible, la surface totale de l'impact atteignant $2 \mathrm{~mm}^{2}$. Le courant primaire étant généralement de $8 \mu \mathrm{A}$ (pour de l'argon extrait à $10 \mathrm{kV}$ ), la densité ionique moyenne du bombardement serait de $4 \mu \mathrm{A} / \mathrm{mm}^{2}$. En fait, cette densité ionique est très inégalement répartie et nous avons alors cherché à connaître sa distribution à l'intérieur du spot primaire.

A cet effet, une petite région du spot primaire est sélectionnée au moyen d'un diaphragme de $0,1 \mathrm{~mm}$ de diamètre, disposé à l'emplacement de l'échantillon et mobile transversalement de manière à décrire toute la largeur du spot. Les ions qui traversent le diaphragme sont recueillis dans un cylindre de Faraday, avec interposition d'une électrode portée à $-100 \mathrm{~V}$, destinée à repousser les électrons secondaires émis par le fond du cylindre. Le courant correspondant est lu sur un nanoampèremètre, tandis que la mesure du courant total s'effectue en reliant à la masse, au travers d'un microampèremètre, le diaphragme et le cylindre qui entoure l'échantillon. Le courant primaire étant de $8 \mu \mathrm{A}$, nous avons ainsi trouvé au centre du spot une densité ionique maximale $i_{\mathrm{m}}=14 \mu \mathrm{A} / \mathrm{mm}^{2}$.

Dans une autre expérience, nous avons bombardé un échantillon composé de quatre plaques de cuivre de $4 \mu \mathrm{m}$ d'épaisseur, empilées sur une pastille d'aluminium, la fin du perçage étant déterminée par l'apparition des ions $\mathrm{Al}+$ parmi les ions secondaires (cette méthode est plus sensible que l'observation de la décroissance simultanée du courant d'ions $\mathrm{Cu}^{+}$). En fait, en raison du décapage préférentiel selon certaines faces cristallines, la dernière plaque n'est pas percée en un seul point, mais présente un grand nombre de petits trous sur une aire relativement étendue. La distribution de la densité ionique s'obtient par la mesure de la surface du trou percé dans chacune des feuilles de cuivre.

Les deux méthodes conduisent à des résultats similaires (fig. 4), et la forme de la distribution est bien approchée par la fonction :

$$
i_{1}(s)=i_{\mathrm{m}}\left(1-\frac{s}{A}\right)^{2}
$$

où $i_{1}(s)$ est la densité ionique sur les bords du trou de surface $s$ et $A$ la surface totale décapée. On constate ainsi qu'à l'intérieur d'un spot de $1 \mathrm{~mm}^{2}$ se trouvent déjà concentrés près de $90 \%$ des ions primaires.

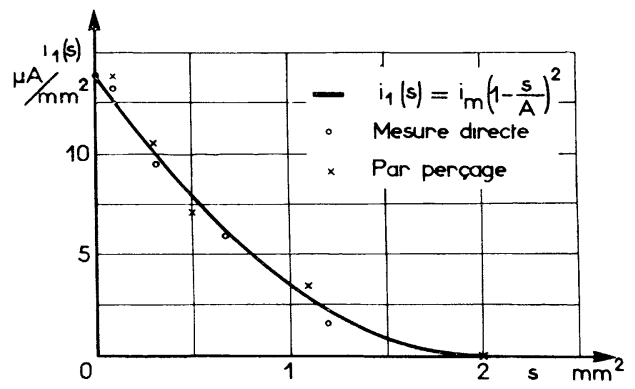

FIG. 4. - Distribution de la densité ionique primaire.

Connaissant la densité ionique maximale $i_{\mathrm{m}}$, nous pouvons maintenant obtenir la valeur du rendement de pulvérisation $S$ d'une substance quelconque, en mesurant le temps $t_{0}$ nécessaire pour en percer une couche d'épaisseur connue $h$, au moyen de la formule :

$$
S=F \frac{\rho_{2}}{M_{2}} \frac{h}{i_{\mathrm{m}} t_{0}} \quad(F: \text { Faraday })
$$

où $\rho_{2}$ et $M_{2}$ sont respectivement la masse volumique et la masse molaire de la substance dont est constituée la cible. Appliquant cette méthode à l'aluminium et au cuivre, nous avons obtenu les valeurs respectives 1,9 et 5,8 atomes par ion, en bon accord avec les valeurs de la littérature [19, 20]. D'autre part, la décroissance du courant secondaire de contrôle $\Delta I(t)$, une fois la tôle percée (fig. 5), suit sensiblement la loi :

$$
\Delta I(t)=\Delta I_{2}\left(\frac{t_{0}}{t}\right)^{3 / 2}
$$

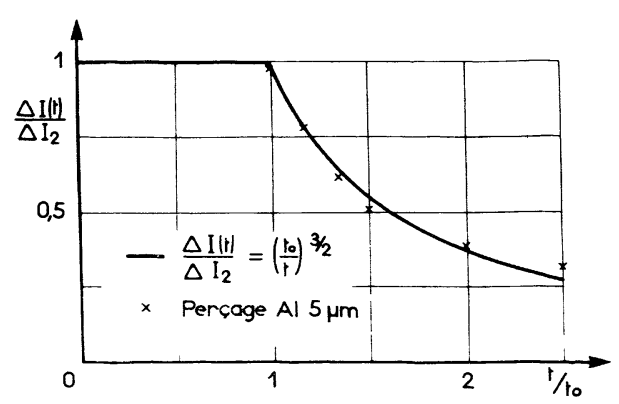

FIg. 5. - Courbe de purçage d'une tôle mince. 
que l'on peut déduire de la forme de la distribution de la densité ionique à l'intérieur du spot primaire ( $\Delta I_{2}$ étant l'intensité secondaire avant perçage). Les écarts à cette loi ne deviennent sensibles qu'après un temps assez long, sans doute à cause du léger balayage du faisceau primaire.

Le bon fonctionnement du système optique de collection des ions secondaires a été contrôlé au moyen d'électrons, en mesurant directement le diamètre du faisceau sur un écran fluorescent escamotable placé à l'entrée du cylindre de Faraday. L'aimant permanent est bien entendu enlevé lors de ces mesures et le cylindre de Faraday calé dans l'axe du dispositif d'extraction et de focalisation, un épais tube de fer armco assurant le blindage magnétique du faisceau. Les électrons sont produits par une cathode à oxydes montée à la place de l'échantillon, l'aire d'émission étant réduite à $1 \mathrm{~mm}^{2}$ par un diaphragme (le filament de tungstène classique produit trop de lumière directe sur l'écran fluorescent et présente par ailleurs une émission trop ponctuelle). Entre la cathode à oxydes et le cylindre qui entoure l'échantillon, ces électrons subissent une première accélération qui définit leur énergie initiale; ils sont ensuite accélérés par le même dispositif que les ions secondaires (au signe des tensions électriques près). Le diamètre mesuré $\mathrm{du}$ faisceau est de $5 \mathrm{~mm}$, nettement inférieur au diamètre de $6 \mathrm{~mm}$ du trou d'entrée du dispositif de collection; ce diamètre ne s'accroît que très peu quand on applique une tension sur la grille de contre-champ. Par contre, les courbes de contrechamp ( $f g .6$, trait fin) présentent deux anomalies, l'une aux basses énergies, l'autre au moment de la

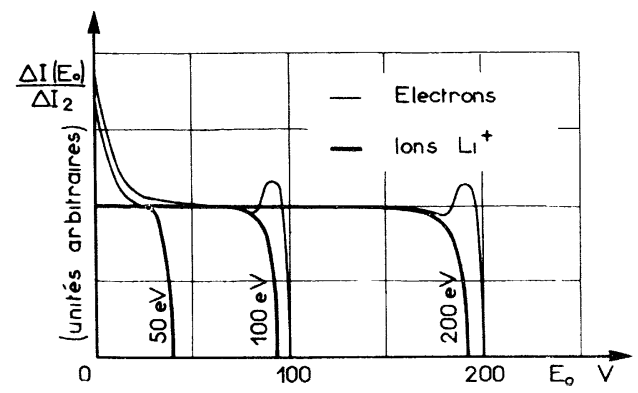

FIG. 6. - Étalonnage du dispositif de contre-champ.

coupure, vraisemblablement dues à des émissions électroniques secondaires parasites ; mais la position même de la coupure est tout à fait correcte.

L'utilisation d'ions, au lieu d'électrons, supprime cette difficulté. Nous avons donc remplacé la cathode à oxydes du dispositif précédent par une petite sphère d'alumino-silicate de lithium chauffée à haute température par un filament de tungstène.
Les ions $\mathrm{Li}^{+}$ainsi émis sont pratiquement monocinétiques [21], mais leur potentiel d'émission est légèrement inférieur au potentiel du filament de chauffage. Les courbes de contre-champ que l'on obtient ( $f$ g. 6, trait fort), ne présentent plus les anomalies observées avec les électrons; par contre la position de la coupure est décalée vers le zéro d'une dizaine de volts. Quoi qu'il en soit, la largeur de la coupure est de l'ordre de $5 \mathrm{eV}$ pour des ions de $30 \mathrm{eV}$ et de $10 \mathrm{eV}$ pour des ions de $100 \mathrm{eV}$ et $300 \mathrm{eV}$. La focalisation devient insuffisante à partir de $400 \mathrm{eV}$ et la largeur de coupure s'étale alors sur près de $50 \mathrm{eV}$ : il en résulte une erreur systématique difficile à estimer dans les mesures faites à ces fortes tensions de contre-champ. Fort heureusement, les énergies moyennes des ions secondaires sont le plus souvent comprises entre 30 et $200 \mathrm{eV}[13,22]$.

L'aimant permanent ayant été remonté et le cylindre de Faraday calé à $45^{\circ}$, nous avons pu vérifier que les caractéristiques du filtrage n'étaient pratiquement pas perturbées par la déflexion magnétique. Les deux isotopes du lithium apparaissent tout juste séparés, ce qui montre bien la faible résolution en masses du système ; on observe aussi deux pics très faibles correspondant à des traces de sodium et de potassium. La dispersion étant de l'ordre de $1 \mathrm{~mm}$ par $100 \mathrm{eV}$ à l'entrée du cylindre de Faraday, il est facile de vérifier que la largeur du faisceau est bien encore voisine de $5 \mathrm{~mm}$, comme avec les électrons.

Conclusion. - L'appareil que nous venons de décrire a déjà donné d'utiles renseignements sur les conditions d'une analyse quantitative des alliages par émission ionique secondaire [23]. La détermination de la distribution angulaire des ions émis par des monocristaux d'aluminium et de cuivre [22] a permis aussi d'établir que, dans le cas du bombardement d'une cible métallique parfaitement propre - c'est-à-dire lorsqu'aucune couche superficielle ne se produit en cours de bombardement par une réaction chimique avec le gaz résiduel dans l'enceinte - les atomes éjectés à la suite de chaînes de collisions focalisées ne sont pas ionisés et ne participent donc pas à l'émission ionique secondaire. La mesure des rendements ioniques et des énergies moyennes d'émission de divers métaux, selon différentes conditions expérimentales, est en cours et fera l'objet de la deuxième partie de ce Mémoire.

Je tiens à exprimer ici mes plus vifs remerciements à M. le Professeur R. Castaing qui m'a proposé cette étude dans son laboratoire et m'a constamment guidé et encouragé dans ces travaux.

Manuscrit reçu le 9 juillet 1966. 


\section{BIBLIOGRAPHIE}

[1] Veksler (V. I.) et Benjaminovitch (M. B.), Soviet Physics, Technical Physics, 1956, 1, 1626.

[2] Honig (R. E.), J. Appl. Physics, 1958, 29, 549.

[3] Bradeey (R. C.), J. Appl. Physics, 1959, 30, 1.

[4] Stanton (H. E.), J. Appl. Physics, 1960, 31, 678.

[5] Castaing (R.), Jouffrey (B.) et Slodzian (G.), C. R., Acad. Sc., 1960, 251, 1010.

[6] Fogel (Ya. M.), Slabospitskil (R. P.) et KarNaukhov (I. M.), Soviet Physics, Technical Physics, 1961, 5, 777 .

[7] White (F. A.), Sheffield (J. C.) et Rourke (F. M.), J. Appl. Physics, 1962, 33, 2915.

[8] Castaing (R.) et Slodzian (G.), J. de Microscopie, 1962, 1, 395.

[9] Srodzian (G.), Thèse Ann. Phyiquue, 1964. 9, 591.

[10] Beske (H. E.), Z. Angew. Physik, 1962, 14, 30.

[11] Smith (A. J.), Marshall (D. J.), Cambey (L. A.) et Micháe (J.), Vacuum, 1964, 14, 263.

[12] Beske (H. E.), Z. Naturforsch., 1964, 19a, 1627.

[13] Veksler (V. I.), Soviet Physics J. E. T. P., 1960, 11, 235 .
[14] Almen (O.) et Nielsen (K. O.), Nucl. Instr., 1957, 1, 302 .

[15] Carlston (C. E.) et Magnuson (G. D.), Rev. Sc. Instr., 1962, 33, 905.

[16] Forst (G.), Z. Physik, 1960, 159, 7.

17] Loeв (H.), Z. Naturforsch., 1961, 16a, 67

[18] Simpsom (J. A.), Rev. Sc. Instr., 1961, 32, 1283.

[19] Rol (P. K.), Fluit (J. M.) et Kistemaker (J.), Physica. 1960, 26, 1000.

[20] Carlston (C. F.), Magnuson (G. D.), Comeaux (A.) et Mahadevan (P.), Phys. Res., 1965, 138, A 759 .

[21] Couchet (G.), Ann. Physique, 1954, 9, 731.

[22] Castaing (R.) et Hennequan (J. F.), C.R. Acad. Sc., 1966, 262, 1008.

[23] Castaing (R.) et Hennequin (J. F.), IVe Congrès International sur l'Optique des Rayons X et la Microanalyse, Hermann, Paris, 1965 (à paraître). 